\title{
Temperature and Size Effects on Radiation-Induced Phase Transformation in Monoclinic Nano-Sized Zirconia
}

\author{
Fengyuan Lu, * Jiaming Zhang, ** Alexandra Navrotsky, ${ }^{* * *}$ R. C. Ewing, ** and Jie \\ Lian* \\ * Department of Mechanical, Aerospace \& Nuclear Engineering, Rensselaer Polytechnic \\ Institute, Troy, NY 12180 \\ ** Departments of Geological Sciences and Materials Science \& Engineering, University \\ of Michigan, Ann Arbor, Michigan 48109-1005 \\ *** NEAT ORU and Peter A. Rock Thermochemistry Laboratory, University of \\ California at Davis, Davis, CA 95616
}

Due to its excellent radiation resistance and thermal-mechanical properties, $\mathrm{ZrO}_{2}$ is considered as a promising candidate material to incorporate actinides for inert matrix fuel and nuclear waste form applications. $\mathrm{ZrO}_{2}$ has polymorphs (cubic, tetragonal, monoclinic) with different physical properties, and undergoes phase transformation under thermal annealing and intensive radiation environments. The phase stability and transformation among different polymorphs greatly affect the material performance used as inert matrix fuel and nuclear waste forms. In this work, we performed energetic beam bombardment experiments using $1 \mathrm{MeV} \mathrm{Kr}^{2+}$ on nano-scale monoclinic $\mathrm{ZrO}_{2}$ to study how temperature and grain size affect phase transformation processes upon irradiation.

Energetic ion beam bombardments were conducted using the IVEM-Tandem facilities at Argonne National Laboratory combining with in-situ transmission electron microscopy (TEM) observation, and the phase transformation among different polymorphs was observed based on selected area electron diffraction patterns (SAED). Figure 1 shows sequences of SAED pattern for monoclinic $\mathrm{ZrO}_{2}$ nanocrystals with average crystal sizes of $17 \mathrm{~nm}$ and $50 \mathrm{~nm}$. A monoclinic-to-tetragonal $(\mathrm{m}-\mathrm{t})$ phase transformation was observed. For $17 \mathrm{~nm} \mathrm{~m}-\mathrm{ZrO}_{2}$, the formation of tetragonal phase started at a dose of 0.7 $\mathrm{dpa}$, and the monoclinic phase is completely eliminated at a dose of $4.2 \mathrm{dpa}$, as evidenced only diffraction rings from the tetragonal phase existing in the diffraction pattern. The $\mathrm{m}$ $\mathrm{t}$ phase transformation was also confirmed by high resolution TEM images (Figure 2). Compared with the smaller-sized $\mathrm{ZrO}_{2}(17 \mathrm{~nm})$ nanocrystals, larger-sized monoclinic $\mathrm{ZrO}_{2}(50 \mathrm{~nm})$ is more resistant to the radiation-induced $\mathrm{m}-\mathrm{t}$ phase transformation, consistent with the fact that the monoclinic phase is thermodynamically more stable at larger size regimes, while tetragonal phase is stabilized towards the smaller end.

For understanding the temperature dependence of radiation-induced $m-t$ phase transformation, $\mathrm{Kr}^{2+}$ irradiation was carried out at different temperatures and temperature dependence of the critical dose driving the formation of tetragonal phase for $17 \mathrm{~nm} \mathrm{ZrO}_{2}$ nanocrystals is shown in Figure 3. Lower temperature is favored in the $\mathrm{m}-\mathrm{t}$ phase transformation process; and the phase transformation process is greatly suppressed at elevated temperature. These results suggest that the radiation-induced defect production and accumulation, specifically oxygen vacancies, are responsible for the $m$-t phase transformation. At elevated temperature, the oxygen vacancies necessary for the formation of tetragonal phase recover due to the dynamic annealing effects. Furthermore, at higher temperature, vacancies may have enough mobility to migrate and annihilate at the surfaces and grain boundaries, and thus, a greater irradiation dose is required to form 
tetragonal phase. The critical temperatures for $\mathrm{m}$-t phase transformation are estimated from the temperature dependence diagrams, above which all of the defects are annealed and the monoclinic phase is stabilized. The critical temperature is around $450 \mathrm{~K}$ and 300 $\mathrm{K}$ for $17 \mathrm{~nm}$ and $50 \mathrm{~nm} \mathrm{~m}-\mathrm{ZrO}_{2}$ respectively. The enhanced stability of monoclinic polymorph at a larger grain size may result from increased self-recombination of oxygen vacancies and interstitials within larger grains. With decreasing grain size less than the characteristic diffusion length of interstitials, the interstitials may be annealed at sinks, leading to the accumulation of oxygen vacancies inside grains and thus easier formation of tetragonal phases. These results suggest that the phase transformation among different polymorphs is a result of interplay among defect production, accumulation inside grains and annihilation at sinks, and both temperature and grain size have a significant impact on the phase stabilities of different $\mathrm{ZrO}_{2}$ polymorphs under intense radiation environments.[1]

[1] This work was supported as part of the Materials Science of Actinides, an Energy Frontier Research Center funded by the U.S. Department of Energy, Office of Science, Office of Basic Energy Sciences under Award Number DE-SC0001089.

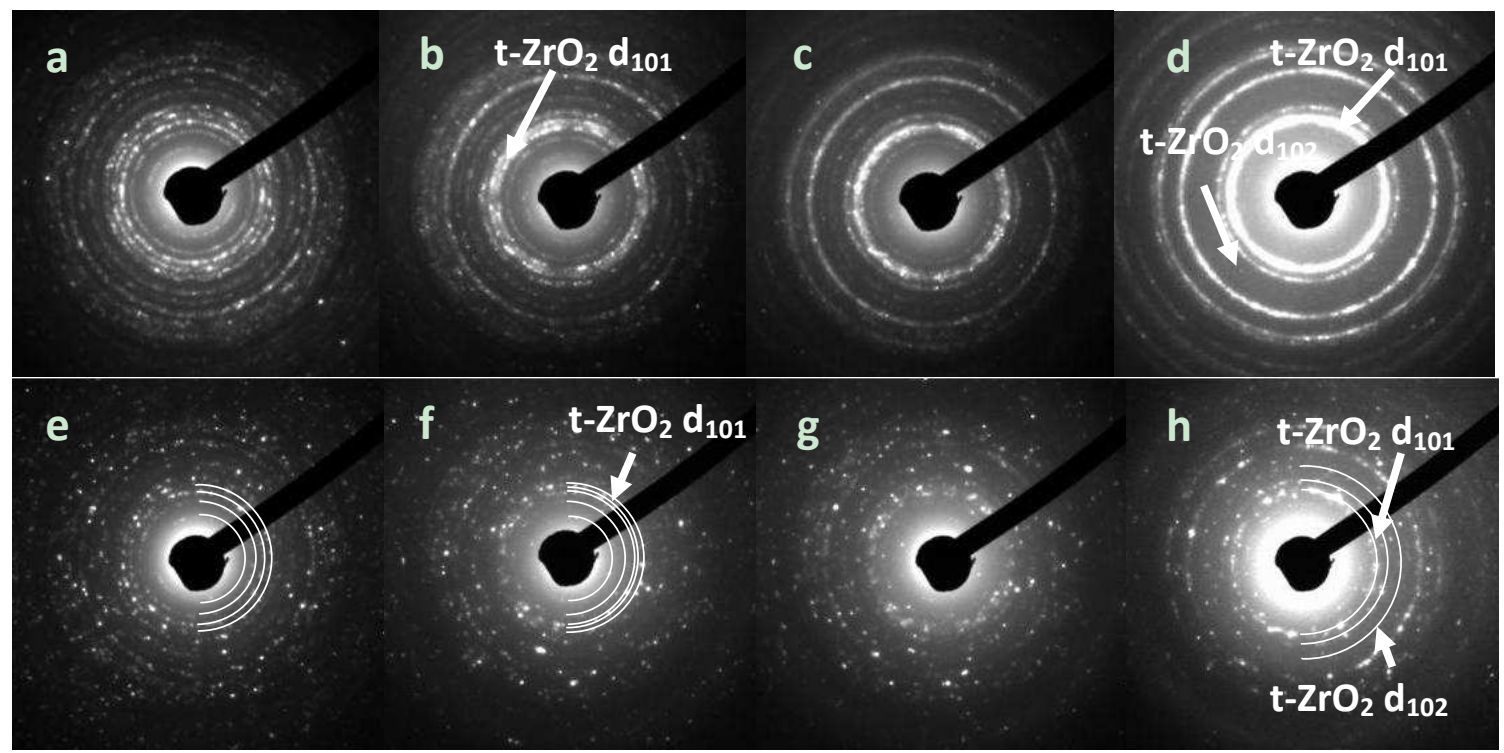

Figure 1. Radiation induced monoclinic-to-tetragonal phase transformation of $\mathrm{ZrO}_{2}$ at 300 Kelvin. For $17 \mathrm{~nm}$ nanocrystals: (a) original monoclinic phase, (b) formation of tetragonal phase at $0.7 \mathrm{dpa}$, (c) progress of phase transformation at $1.7 \mathrm{dpa}$, (d) completion of m-t phase transformation at $4.2 \mathrm{dpa}$. For $50 \mathrm{~nm}$ nanocrystals: (e) original monoclinic phase, (f) formation of tetragonal phase at 2.6 $\mathrm{dpa},(\mathrm{g})$ progress of phase transformation at $4.6 \mathrm{dpa}$, (h) monoclinic residue still exists at $8.3 \mathrm{dpa}$.

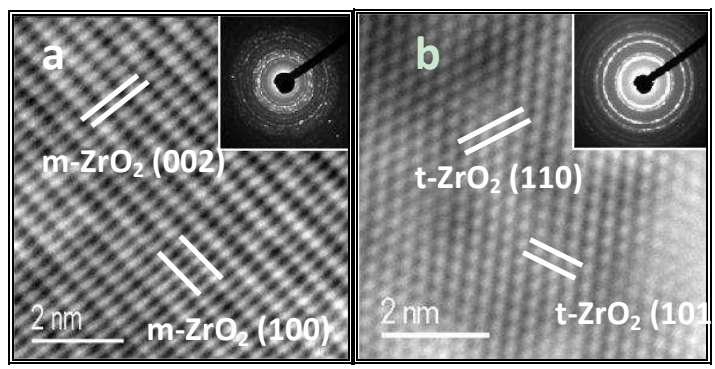

Figure 2. HRTEM images of $17 \mathrm{~nm} \mathrm{ZrO}_{2}$ nanocrystals before and after irradiation: (a) monoclinic structure before irradiation, zone axis [010], (b) tetragonal structure after irradiation, zone axis $[\mathbf{1 1 1}]$.

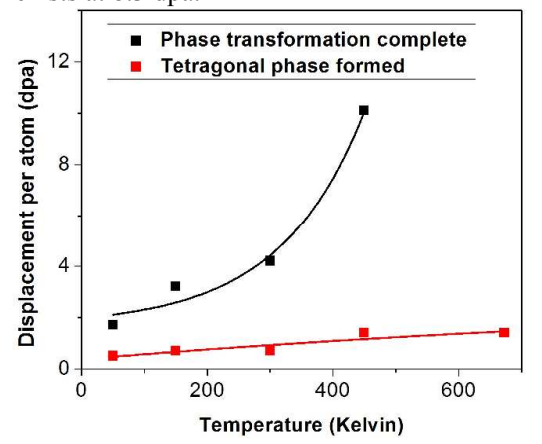

Figure 3. Temperature dependence of irradiation induced $\mathrm{m}$-t phase transformation. Black dots/line: tetragonal phase formation dose. Red dots/line: phase transformation completion dose (monoclinic phase gone). 